\title{
Effects of Heat Transfer and an Endoscope on Peristaltic Flow of a Fractional Maxwell Fluid in a Vertical Tube
}

\author{
H. Rachid \\ Department of Physics, Faculty of Sciences Ain Chock, University of Hassan II, 5366 Casablanca, Morocco \\ Correspondence should be addressed to H. Rachid; rhassan2@yahoo.fr
}

Received 29 May 2015; Revised 23 September 2015; Accepted 30 September 2015

Academic Editor: Changbum Chun

Copyright (C) 2015 H. Rachid. This is an open access article distributed under the Creative Commons Attribution License, which permits unrestricted use, distribution, and reproduction in any medium, provided the original work is properly cited.

We investigate the unsteady peristaltic transport of a viscoelastic fluid with fractional Maxwell model through two coaxial vertical tubes. This analysis has been carried under low Reynolds number and long wavelength approximations. Analytical solution of the problem is obtained by using a fractional calculus approach. The effects of Grashof number, heat parameter, relaxation time, fractional time derivative parameter, amplitude ratio, and the radius ratio on the pressure gradient, pressure rise, and the friction forces on the inner and outer tubes are graphically presented and discussed.

\section{Introduction}

Peristaltic transport is a mechanism of pumping fluids in tubes when progressive wave of area contraction or expansion propagates along the length on the boundary of a distensible tube containing fluid. In the physiological process, peristalsis or the blood flow is the major applications of this transport phenomenon. The contractions and relaxations of the walls propel the fluid. The first investigation to study the peristaltic pumping was by Latham [1]. The peristaltic transport of a Newtonian fluid at long wavelengths at low Reynolds number has been studied with particles suspended by Kaimal [2] or without particles by Shapiro et al. [3] and by Mishra and Rao [4]. The non-Newtonian effects of fluids on the peristaltic transport have been studied for a third-grade fluid by Ali et al. [5], for a Jeffrey fluid by Abd-Alla et al. [6], for a Maxwell model by Rachid and Ouazzani [7], for a Herschel-Bulkley fluid by Medhavi [8], or for a power-law fluid by Shukla and Gupta [9]. The effect of an endoscope on peristaltic flow for a Newtonian fluid through a nonuniform annulus has been analyzed by Mekheimer [10]. This effect has been also studied for a Johnson Segalman fluid by Akbar and Nadeem [11] or for a Maxwell fluid by Husseny et al. [12]. Recently, the peristaltic transport of non-Newtonian fluids has gained considerable interest because of its applications in industry and biology.
Mathematically, the non-Newtonian fluids have a nonlinear relationship between the stress and the rate of strain. It is difficult to propose a single model which can exhibit all the properties of non-Newtonian fluids. To describe the viscoelastic properties of such fluids recently, constitutive equations with ordinary and fractional time derivatives have been introduced. Fractional calculus has proved to be very successful in the description of constitutive relations of viscoelastic fluids. The starting point of the fractional derivative model of viscoelastic fluids is usually a classical differential equation which is modified by replacing the time derivative of an integral order with fractional order and may be formulated both in the Riemann-Liouville and in the Caputo sense [13]. This generalization allows one to define precisely noninteger order integrals or derivatives and this plays an important role in studying the valuable tool of viscoelastic properties. In recent years and considering the relevance of fractional models of viscoelastic fluids, a number of articles have addressed unsteady flows of viscoelastic fluids with the fractional Maxwell model [14, 15], fractional second-grade fluid [16, 17], fractional Oldroyd-B model $[18,19]$, and fractional Burgers model [20]. For example, Tripathi [21] studied a mathematical model based on viscoelastic fractional Oldroyd-B model for the peristaltic flow of chyme in small intestine, which is assumed to be in the form of an inclined cylindrical tube. 
The peristaltic transport for a fractional second-grade model through a cylindrical tube has been investigated by Tripathi [22]. Rathod and Channakote [23] analyzed the interaction of heat transfer on peristaltic pumping of a fractional secondgrade fluid through a vertical cylindrical tube by using Caputo's definition. For the fractional Maxwell model, Tripathi et al. [24] studied the peristaltic flow through a channel where the homotopy perturbation method (HPM) and Adomian decomposition method (ADM) are used. For the fractional Burger's model Tripathi et al. [25] studied the influence of slip condition on peristaltic transport by using a homotopy analysis method (HAM). This generalized model of fluid has been investigated for an infinite oscillating plate by Khan et al. [26], for an orthogonal rheometer by Siddiqui et al. [27] or between concentric cylinders by Shah [28]. Heat transfer analysis is one of the principle axes of research in chemical engineering, but nowadays due to its wide applications in biofluid mechanics it has great interaction with peristaltic motion. The effect of heat transfer on peristaltic transport of a Newtonian fluid through a porous medium in a vertical tube under the effect of a magnetic field has been studied by Vasudev et al. [29]. This effect on peristaltic flow with slip condition and variable viscosity in an asymmetric channel has been analyzed by Hayat and Abbasi [30]. Hayat et al. [31] investigated the effects of heat and mass transfer and wall properties on the peristalsis in a power-law fluid.

The aim of this paper is to analytically study the effect of heat transfer on peristaltic flow of a viscoelastic fluid with fractional Maxwell model in the gap between two coaxial vertical tubes. The influence of different physical parameters on the pressure gradient, pressure rise, and the fictional forces has been graphically shown and discussed.

\section{Formulation and Analysis}

Consider the peristaltic flow of a viscoelastic fluid with fractional Maxwell model through the gap between two coaxial vertical tubes. The inner tube is rigid (endoscope) maintained at a temperature $T_{1}$, and the outer tube has a sinusoidal wave traveling down its wall and it is exposed to temperature $T_{0}$ as seen in Figure 1.

In a cylindrical coordinate system $(\bar{R}, \bar{Z})$, the equations for the tube walls in their dimensional form are given by

$$
\begin{aligned}
& \bar{r}_{1}=a_{1}, \\
& \bar{r}_{2}=a_{2}+b \sin \left(\frac{2 \pi}{\lambda}(\bar{Z}-c \bar{t})\right),
\end{aligned}
$$

where $a_{1}$ is the radius of the endoscope, $a_{2}$ is the average radius, $b$ is the amplitude of the wave, $\lambda$ is the wavelength, and $c$ is the wave speed of the outer tube.

The equation of the fractional Maxwell fluid is given by

$$
\left(1+{\overline{\lambda_{1}}}^{\alpha_{1}} \widetilde{D}_{\bar{t}}^{\alpha_{1}}\right) \bar{S}=\mu \dot{\gamma}
$$

with $\bar{t}, \bar{S}, \dot{\gamma}$, and $\mu$ being the time, shear stress, rate of shear strain, and the viscosity, respectively. $\overline{\lambda_{1}}$ is the relaxation time and $\alpha_{1}$ is the fractional time derivative parameter such that

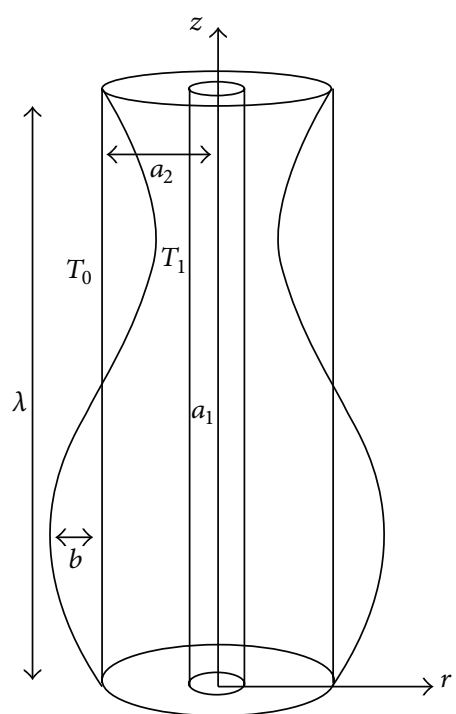

FigURE 1: Geometry of the problem.

$0 \leq \alpha_{1} \leq 1 . \widetilde{D}_{t}^{\alpha_{1}}$ is the upper convected fractional derivative defined by

$$
\widetilde{D}_{\bar{t}}^{\alpha_{1}}(\bar{S})=D_{\bar{t}}^{\alpha_{1}}(\bar{S})+(\bar{V} \cdot \nabla)(\bar{S})-\bar{L}(\bar{S})-(\bar{S}) \bar{L}^{T}
$$

in which

$$
\dot{\gamma}=(\nabla \bar{V})+(\nabla \bar{V})^{T}
$$

and $D_{\bar{t}}^{\alpha_{1}}=\partial_{\bar{t}}^{\alpha_{1}}$ is the fractional differentiation operator of order $\alpha_{1}$ with respect to $t$ and may be defined as [32]

$$
D_{t}^{\alpha_{1}} f(t)=\frac{1}{\Gamma\left(1-\alpha_{1}\right)} \frac{d}{d t} \int_{0}^{t} \frac{f(\xi)}{(t-\xi)^{\alpha_{1}}} d \xi, \quad 0 \leq \alpha_{1} \leq 1 .
$$

Here $\Gamma(\cdot)$ denotes the Gamma function.

We choose a cylindrical coordinate system $(\bar{r}, \bar{z})$ where $\bar{z}$ axis is the longitudinal direction and the $\bar{r}$-axis is transverse to it.

The equations of motion of the flow for an incompressible fluid are given by

$$
\begin{aligned}
& \rho\left[\frac{\partial \bar{u}}{\partial \bar{t}}+\bar{u} \frac{\partial \bar{u}}{\partial \bar{r}}+\bar{w} \frac{\partial \bar{u}}{\partial \bar{z}}\right]=-\frac{\partial \bar{p}}{\partial \bar{r}}+\frac{1}{\bar{r}} \frac{\partial}{\partial \bar{r}}\left(\bar{r} \bar{S}_{\bar{r} \bar{r}}\right)+\frac{\partial \bar{S}_{\overline{r z}}}{\partial \bar{z}}, \\
& \rho\left[\frac{\partial \bar{w}}{\partial \bar{t}}+\bar{u} \frac{\partial \bar{w}}{\partial \bar{r}}+\bar{w} \frac{\partial \bar{w}}{\partial \bar{z}}\right] \\
& =-\frac{\partial \bar{p}}{\partial \bar{z}}+\frac{1}{\bar{r}} \frac{\partial}{\partial \bar{r}}\left(\bar{r} \bar{S}_{\overline{r z}}\right)+\frac{\partial \bar{S}_{\overline{z z}}}{\partial \bar{z}}+\rho g \alpha\left(\bar{T}-T_{0}\right), \\
& \rho c_{p}\left[\bar{u} \frac{\partial \bar{T}}{\partial \bar{r}}+\bar{w} \frac{\partial \bar{T}}{\partial \bar{z}}\right]=k\left[\frac{\partial^{2} \bar{T}}{\partial \bar{r}^{2}}+\frac{1}{\bar{r}} \frac{\partial \bar{T}}{\partial \bar{r}}+\frac{\partial^{2} \bar{T}}{\partial \bar{z}^{2}}\right]+Q_{0}, \\
& \frac{\partial \bar{u}}{\partial \bar{r}}+\frac{\bar{u}}{\bar{r}}+\frac{\partial \bar{w}}{\partial \bar{z}}=0,
\end{aligned}
$$

where $\rho$ is the fluid density, $\bar{T}$ is the temperature, $\alpha$ is the coefficient of linear thermal expansion of the fluid, $k$ denotes 
thermal conductivity, $c_{p}$ denotes specific heat at constant pressure, $Q_{0}$ is the constant heat addition/absorption, $\bar{u}$ and $\bar{w}$ are the velocity components in the wave frame, and $\bar{p}$ is the pressure.

We assume that the extra stress $\bar{S}$ depends on $r$ and $t$ only. After using the initial condition $\bar{S}(\bar{t}=0)$, (2)-(4) yield $\bar{S}_{\overline{r r}}=$ $\bar{S}_{\overline{z z}}=\bar{S}_{\bar{r} \bar{\theta}}=\bar{S}_{\bar{\theta} \bar{z}}=0$ and

$$
\left(1+\bar{\lambda}_{1}^{\alpha_{1}} \frac{\partial^{\alpha_{1}}}{\partial \bar{t}^{\alpha_{1}}}\right) \bar{S}_{\overline{r z}}=\mu \frac{\partial \bar{w}}{\partial \bar{r}}
$$

where $\bar{S}_{\bar{r} \bar{z}}$ is the tangential stress.

For carrying out further analysis, we introduce the following dimensionless parameters:

$$
\begin{aligned}
& z=\frac{\bar{z}}{\lambda} ; \\
& Z=\frac{\bar{Z}}{\lambda} ; \\
& r_{1}=\frac{\bar{r}_{1}}{a_{2}} ; \\
& r_{2}=\frac{\bar{r}_{2}}{a_{2}} ; \\
& R=\frac{\bar{R}}{a_{2}} ; \\
& t=\frac{c \bar{t}}{\lambda} ; \\
& u=\frac{\lambda \bar{u}}{a_{2} c} ; \\
& U=\frac{\lambda \bar{U}}{a_{2} c} ; \\
& w=\frac{\bar{w}}{c} ; \\
& W=\frac{\bar{W}}{c} ; \\
& \lambda_{1}=\frac{\overline{c \lambda_{1}}}{\lambda} ; \\
& \varepsilon=\frac{a_{1}}{a_{2}}, \\
& p=\frac{a_{2}^{2} \bar{p}}{\mu \lambda c} ; \\
& \theta=\frac{\bar{T}-T_{0}}{T_{1}-T_{0}} ; \\
& Q=\frac{\bar{Q}}{\pi c a_{2}^{2}} ; \\
& \delta=\frac{a_{2}}{\lambda} ;
\end{aligned}
$$

$$
\begin{aligned}
\operatorname{Pr} & =\frac{\mu c_{p}}{k} ; \\
\operatorname{Re} & =\frac{\rho c a_{2}}{\mu_{0}} ; \\
\beta & =\frac{a_{2}^{2} Q_{0}}{k\left(T_{1}-T_{0}\right)} ; \\
\mathrm{Gr} & =\frac{\rho g \alpha a_{2}^{3}\left(T_{1}-T_{0}\right)}{\mu},
\end{aligned}
$$

where $\varepsilon$ is the radius ratio, $\delta$ is the wave number, $\mathrm{Re}$ is the Reynolds number, Pr is the Prandtl number, Gr is the Grashof number, $\beta$ is the nondimensional heat source/sink parameter, and $\phi$ is the amplitude ratio with $0<\phi<1-\varepsilon$.

Using the above nondimensional quantities and under the assumptions of long wavelength approximation (i.e., $\delta \ll 1$ or $\lambda \gg a_{2}$ ) and the low Reynolds number (i.e., $\operatorname{Re} \rightarrow 0$ ), (6) yield

$$
\begin{aligned}
\frac{\partial p}{\partial r} & =0, \\
\left(1+\lambda_{1}^{\alpha_{1}} D_{t}^{\alpha_{1}}\right)\left(\frac{\partial p}{\partial z}-\mathrm{Gr} \theta\right) & =\left(\frac{\partial^{2} w}{\partial r^{2}}+\frac{1}{r} \frac{\partial w}{\partial r}\right), \\
\frac{\partial u}{\partial r}+\frac{u}{r}+\frac{\partial w}{\partial z} & =0, \\
\frac{\partial^{2} \theta}{\partial r^{2}}+\frac{1}{r} \frac{\partial \theta}{\partial r}+\beta & =0 .
\end{aligned}
$$

The boundary conditions are

$$
\begin{aligned}
& w=-1 ; \\
& \theta=1 \\
& \text { at } r=r_{1}=\varepsilon \text {, } \\
& w=-1 ; \\
& \theta=0
\end{aligned}
$$

where $r_{2}=1+\phi \sin (2 \pi z)$ is the dimensionless equation of the outer tube radius in the wave frame.

Integrating (12) with the boundary conditions (13), we find the dimensionless temperature as follows:

$$
\theta=\frac{\ln \left(r / r_{2}\right)}{\ln \left(\varepsilon / r_{2}\right)}+\frac{\beta}{4} \frac{\left(\varepsilon^{2} \ln \left(r / r_{2}\right)-r_{2}^{2} \ln (r / \varepsilon)\right)}{\ln \left(\varepsilon / r_{2}\right)}-\frac{\beta}{4} r^{2} \text {. }
$$

Use the transformations between the laboratory and the wave frames, in the dimensionless form, given by

$$
\begin{aligned}
& z=Z-t ; \\
& r=R ; \\
& u=U ; \\
& w=W-1,
\end{aligned}
$$


where $\bar{U}$ and $\bar{W}$ are the velocity components in the fixed frame.

Substituting (14) in (10) and using the same boundary conditions, we obtain the velocity profile of the fluid as

$$
\begin{aligned}
W & =\frac{1}{4} f\left(r^{2}-a_{21} \ln (r)+a_{25}\right) \frac{\partial p}{\partial z}+\frac{1}{64} f \mathrm{Gr}\left[\beta r^{4}\right. \\
& \left.+4\left(\beta a_{24} \ln \left(\frac{r}{\varepsilon}\right)-a_{41} \ln \left(\frac{r}{r_{2}}\right)+a_{42}\right) r^{2}\right]+\frac{1}{64} \\
& \cdot f \mathrm{Gr}_{43},
\end{aligned}
$$

where $f(t)=1+\lambda_{1}^{\alpha_{1}} D_{t}^{\alpha_{1}}=f$.

Using the definition of the fractional differential operator (5) we find the expression of $f$ as follows:

$$
f=1+\lambda_{1}^{\alpha_{1}} \frac{t^{-\alpha_{1}}}{\Gamma\left(1-\alpha_{1}\right)} .
$$

The volume rate of flow in the fixed coordinate system $(R$, $Z$ ) is given as

$$
Q(Z, t)=2 \int_{\varepsilon}^{r_{2}} W R d R=f\left(a_{61} \frac{\partial p}{\partial z}+a_{62}\right) .
$$

Using the transformation (15) we find

$$
Q(Z, t)=q+r_{2}^{2}-\varepsilon^{2},
$$

where $q$ is the volume flow rate in the moving coordinate system given by

$$
q=2 \int_{\varepsilon}^{r_{2}} w r d r
$$

The time-averaged flow rate is defined by

$$
Q=\int_{0}^{1} Q(Z, t) d t=q+1+\frac{\phi^{2}}{2}-\varepsilon^{2} .
$$

Eliminating the flow rate $q$ between (19) and (21) we find

$$
Q(Z, t)=Q+r_{2}^{2}-1-\frac{\phi^{2}}{2} \text {. }
$$

From (18) and (22) we obtain the pressure gradient as follows:

$$
\frac{\partial p}{\partial z}=\frac{1}{a_{61}}\left[\left(\frac{Q+r_{2}^{2}-1-\phi^{2} / 2}{f}\right)-a_{62}\right] .
$$

\section{The Pumping Characteristics}

The pressure rise $\Delta p$ and the frictional force $F_{\lambda}$ at the walls of the inner and outer tubes, in the nondimensional form, are given by

$$
\begin{aligned}
\Delta p & =\int_{0}^{1} \frac{\partial p}{\partial z} d z, \\
F^{(i)} & =\int_{0}^{1} \varepsilon^{2}\left(-\frac{\partial p}{d z}\right) d z, \\
F^{(o)} & =\int_{0}^{1} r_{2}^{2}\left(-\frac{\partial p}{d z}\right) d z .
\end{aligned}
$$

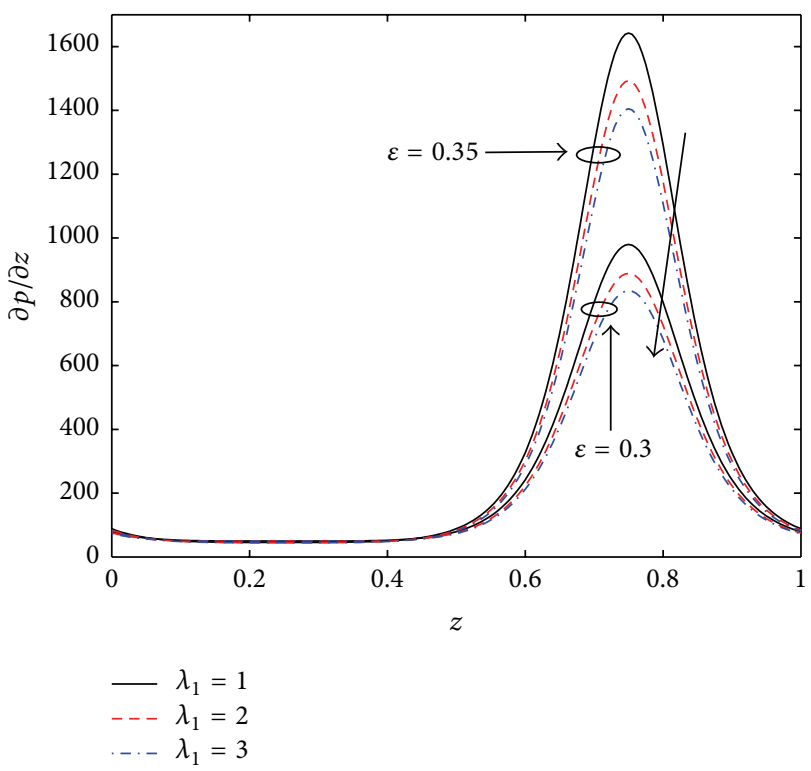

FIGURE 2: Pressure gradient $\partial p / \partial z$ versus axial distance $z$ for different values of the relaxation time $\lambda_{1}$ and radius ratio $\varepsilon$ with $Q=-2$, $\mathrm{Gr}=3, \alpha_{1}=0.4, \beta=10, \phi=0.4$, and $t=1$.

\section{Results and Discussions}

We note that the fractional Maxwell model reduces to a Newtonian fluid when $\overline{\lambda_{1}}=0$.

The analytical expressions for the pressure gradient $\partial p /$ $\partial z$, the pressure rise $\Delta p$, and the frictional forces on the inner and outer tubes, respectively, $F^{(i)}$ and $F^{(o)}$, are derived in the previous sections. In order to compute these physical quantities with respect to interest parameters of problem we observe that the integrals in (24) are not integrable in the closed form. They are evaluated numerically using a mathematics software.

Pressure gradient $\partial p / \partial z$ against the axial distance $z$ on one wavelength for different given values of the Grashof number $\mathrm{Gr}$, the heat parameter $\beta$, the relaxation time $\lambda_{1}$, the fractional time derivative parameter $\alpha_{1}$, the amplitude ratio $\phi$, and the radius ratio $\varepsilon$ is plotted in Figures 2-4. These figures show that, in the wider part of the tube $(z \in[0,0.5]), \partial p / \partial z$ is relatively small, where the flow can easily pass without giving any large pressure gradient. However, in the narrow part $(z \in[0.5,1])$ a much larger pressure gradient is required to maintain the same flux to pass it, especially for the narrowest position near $z=0.75$. Moreover the maximum of the pressure gradient increases with increasing $\mathrm{Gr}, \phi, \varepsilon$, and $\alpha_{1}$ while it decreases with the increase in $\lambda_{1}$ and $\beta$.

The effects of various parameters on the pressure rise $\Delta p$ versus the time-averaged flow rate $Q$ are shown in Figures 57. It is known that we have the following peristaltic regions, pumping region $(\Delta p>0)$, free-pumping region $(\Delta p=0)$, and copumping region $(\Delta p<0)$. These figures show a linear relationship between $\Delta p$ and $Q$ in all the regions; that is, the pressure rise decreases with increasing the time-averaged flow rate. From Figure 5 we observe that the time-averaged flow rate $Q$ increases with increasing $\mathrm{Gr}$ while it decreases with the increase in $\beta$ in all the regions. In addition, $\Delta p$ and 


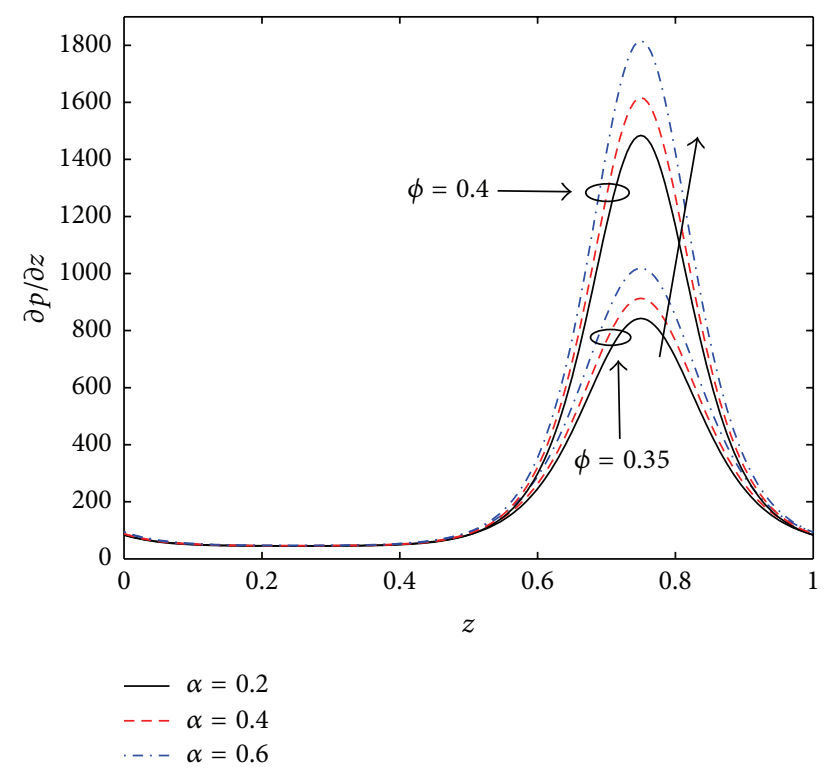

FIGURE 3: Pressure gradient $\partial p / \partial z$ versus axial distance $z$ for different values of the parameter $\alpha_{1}$ and amplitude ratio $\phi$ with $Q=-2$, $\mathrm{Gr}=3, \varepsilon=0.35, \beta=10, \lambda_{1}=1$, and $t=1$.

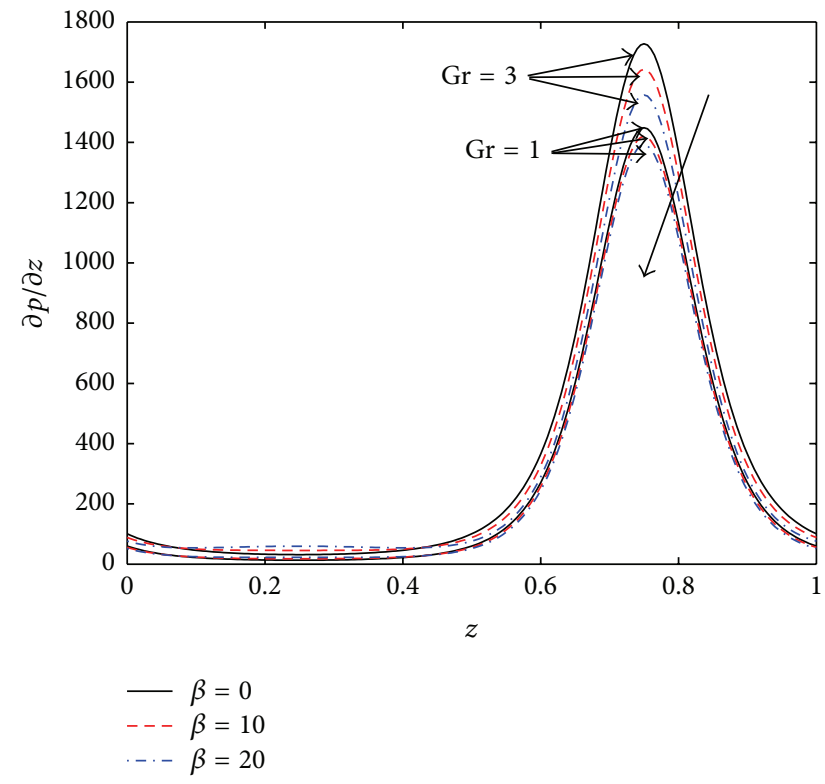

FIgURE 4: Pressure gradient $\partial p / \partial z$ versus axial distance $z$ for different values of the heat parameter $\beta$ and the Grashof number Gr with $Q=-2, \lambda_{1}=1, \alpha_{1}=0.4, \varepsilon=0.35, \phi=0.4$, and $t=1$.

the flow rate $Q$ and the pumping increase with increasing $\mathrm{Gr}$ while they decrease with the increase in $\beta$. Figures 6-7 show that for different values of $\varepsilon, \lambda_{1}, \phi$, and $\alpha_{1}$ the curves are intersecting in the pumping region. In addition, for a given value of the pressure rise $\Delta p$ we observe that in both the freepumping and copumping regions the time-averaged flow rate $Q$ increases with increasing $\lambda_{1}$ while it decreases with the increase in $\alpha_{1}$. The same figures show that $Q$ decreases in

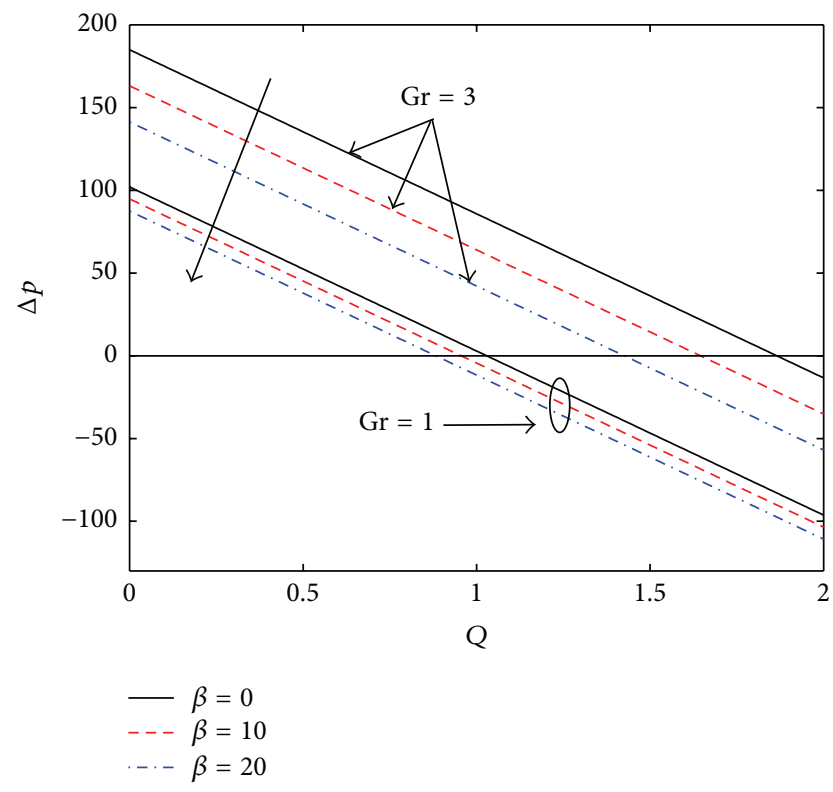

FIgURE 5: Pressure rise $\Delta p$ versus the time-averaged flow rate $Q$ for different values of the heat parameter $\beta$ and the Grashof number $\mathrm{Gr}$ with $\lambda_{1}=1, \alpha_{1}=0.4, \varepsilon=0.35, \phi=0.4$, and $t=1$.

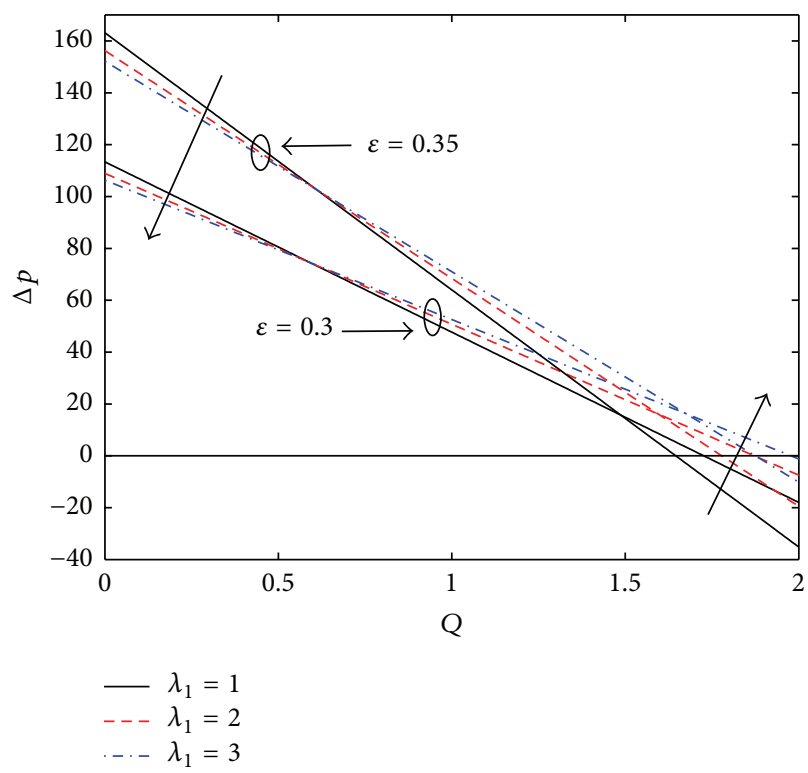

FIGURE 6: Pressure rise $\Delta p$ versus the time-averaged flow rate $Q$ for different values of the relaxation time $\lambda_{1}$ and radius ratio $\varepsilon$ with $\mathrm{Gr}=$ $3, \alpha_{1}=0.4, \beta=10, \phi=0.4$, and $t=1$.

the free-pumping and copumping regions with increasing $\varepsilon$ and $\phi$.

In Figures 8-13 we plotted the frictional forces $F^{(i)}$ and $F^{(o)}$ on the inner and outer tubes, respectively. These figures show that $F^{(i)}$ and $F^{(o)}$ have an opposite behavior compared to the pressure rise $\Delta p$ versus the physical parameters. In addition the frictional force on the outer tube $F^{(o)}$ is greater than the frictional force on the outer tube $F^{(i)}$. 


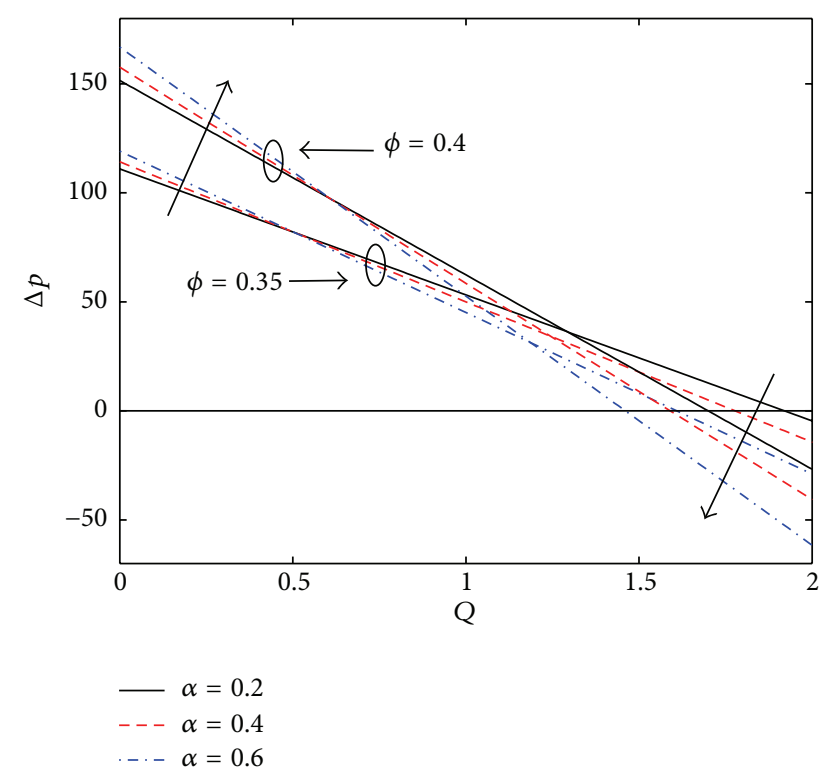

FIGURE 7: Pressure rise $\Delta p$ versus the time-averaged flow rate $Q$ for different values of the parameter $\alpha_{1}$ and amplitude ratio $\phi$ with $\mathrm{Gr}=$ $3, \varepsilon=0.35, \beta=10, \lambda_{1}=1$, and $t=1$.

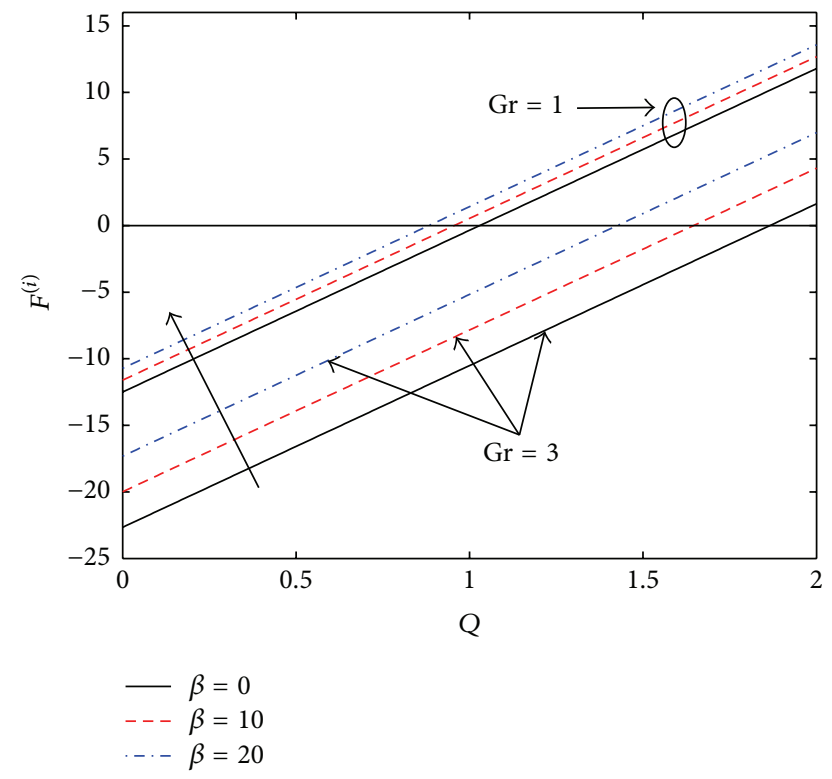

FIGURE 8: Frictional force on the inner tube $F^{(i)}$ versus the timeaveraged flow rate $Q$ for different values of heat parameter $\beta$ and the Grashof number Gr with $\lambda_{1}=1, \alpha_{1}=0.4, \varepsilon=0.35, \phi=0.4$, and $t=1$.

\section{Conclusions}

We have analytically studied the effect of heat transfer on peristaltic flow of fractional Maxwell model in the gap between two vertical coaxial tubes. The problem is simplified under the assumptions of long wavelength approximation and low Reynolds number. Analytical solution of problem is obtained by using a fractional calculus approach. The pressure gradient, pressure rise, and frictional forces on the endoscope and on the outer tube are discussed with the physical

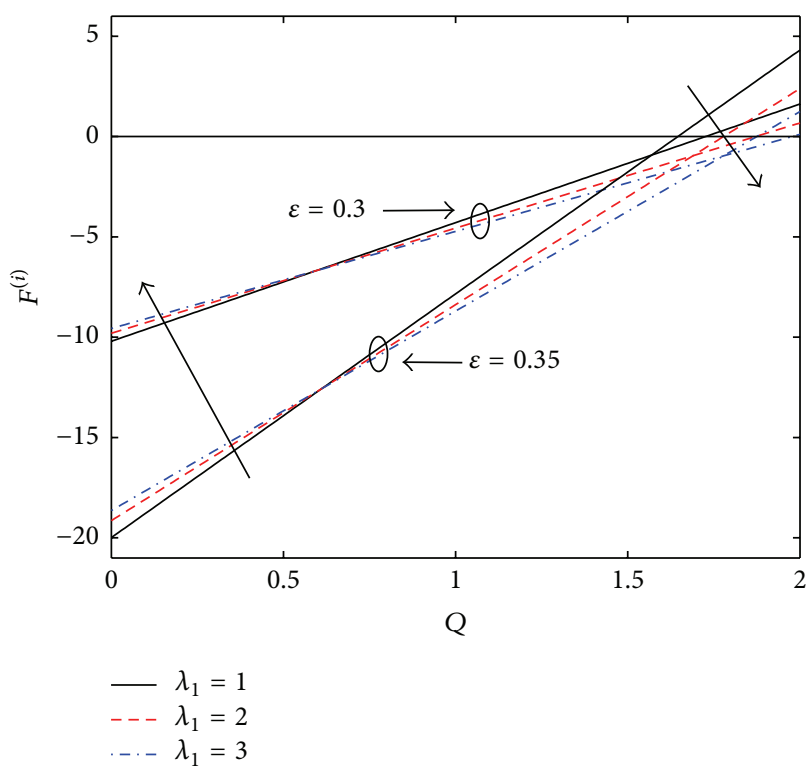

FIgURE 9: Frictional force on the inner tube $F^{(i)}$ versus the timeaveraged flow rate $Q$ for different values of the relaxation time $\lambda_{1}$ and radius ratio $\varepsilon$ with $\mathrm{Gr}=3, \alpha_{1}=0.4, \beta=10, \phi=0.4$, and $t=1$.

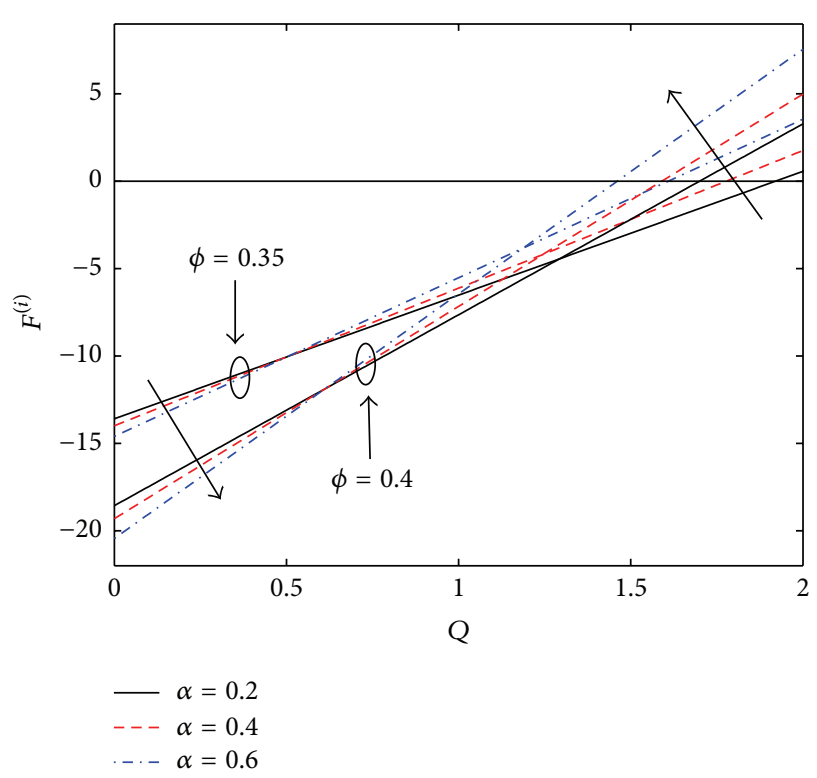

FIGURE 10: Frictional force on the inner tube $F^{(i)}$ versus the timeaveraged flow rate $Q$ for different values of the parameter $\alpha_{1}$ and amplitude ratio $\phi$ with $\mathrm{Gr}=3, \varepsilon=0.35, \beta=10, \lambda_{1}=1$, and $t=1$.

parameters, Grashof number Gr, the heat parameter $\beta$, the relaxation time $\lambda_{1}$, the fractional time derivative parameter $\alpha_{1}$, the amplitude ratio $\phi$, and the radius ratio $\varepsilon$. The graphical solutions have shown the following:

(1) The maximum of the pressure gradient $\partial p / \partial z$ increases with increasing $\mathrm{Gr}, \phi, \varepsilon$, and $\alpha_{1}$ while it decreases with the increase in $\lambda_{1}$ and $\beta$.

(2) The time-averaged flow rate $Q$ increases with increasing $\mathrm{Gr}$ while it decreases with the increase in $\beta$ in 


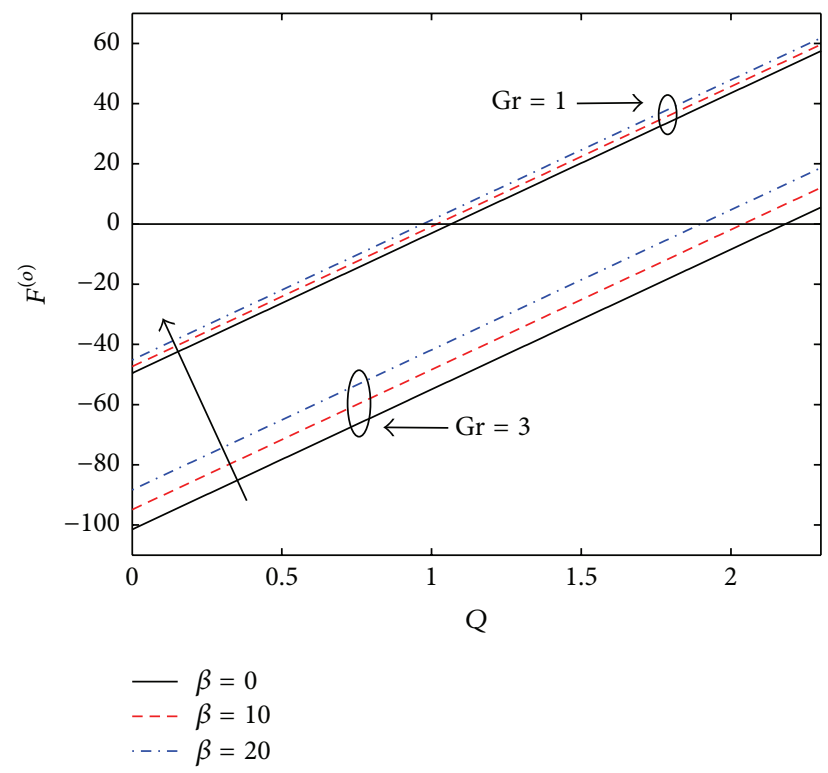

FIGURE 11: Frictional force on the outer tube $F^{(o)}$ versus the timeaveraged flow rate $Q$ for different values of heat parameter $\beta$ and the Grashof number Gr with $\lambda_{1}=1, \alpha_{1}=0.4, \varepsilon=0.35, \phi=0.4$, and $t=1$.

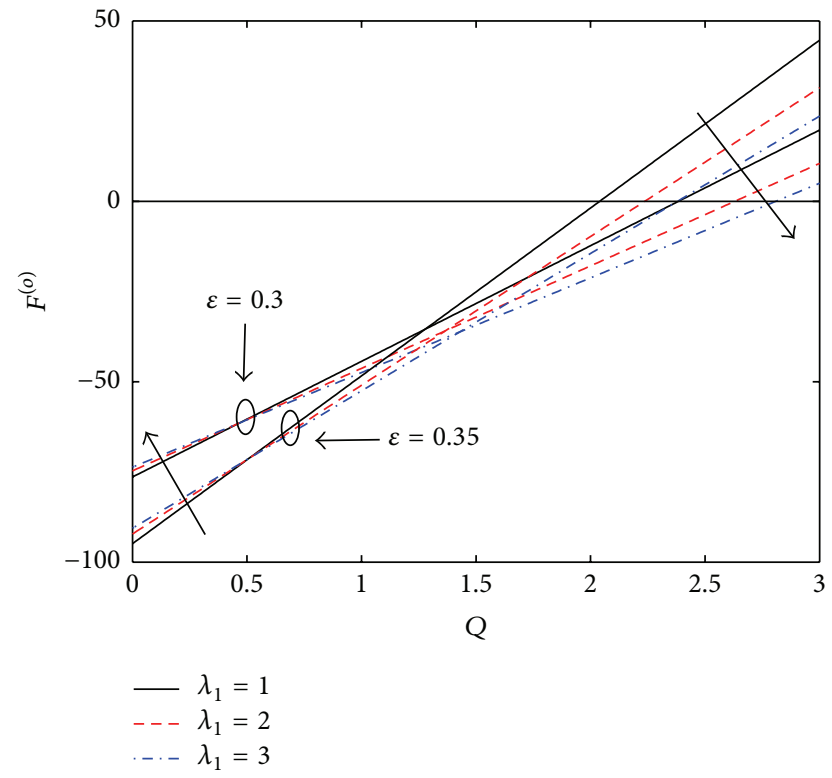

FIGURE 12: Frictional force on the outer tube $F^{(o)}$ versus the timeaveraged flow rate $Q$ for different values of the relaxation time $\lambda_{1}$ and radius ratio $\varepsilon$ with $\mathrm{Gr}=3, \alpha_{1}=0.4, \beta=10, \phi=0.4$, and $t=1$.

all the regions of pumping for a given value of the pressure rise $\Delta p$.

(3) $Q$ increases with the increase in $\lambda_{1}$ in both the freepumping and co-pumping regions while we observe an opposite behavior versus $\alpha_{1}$ in these two regions.

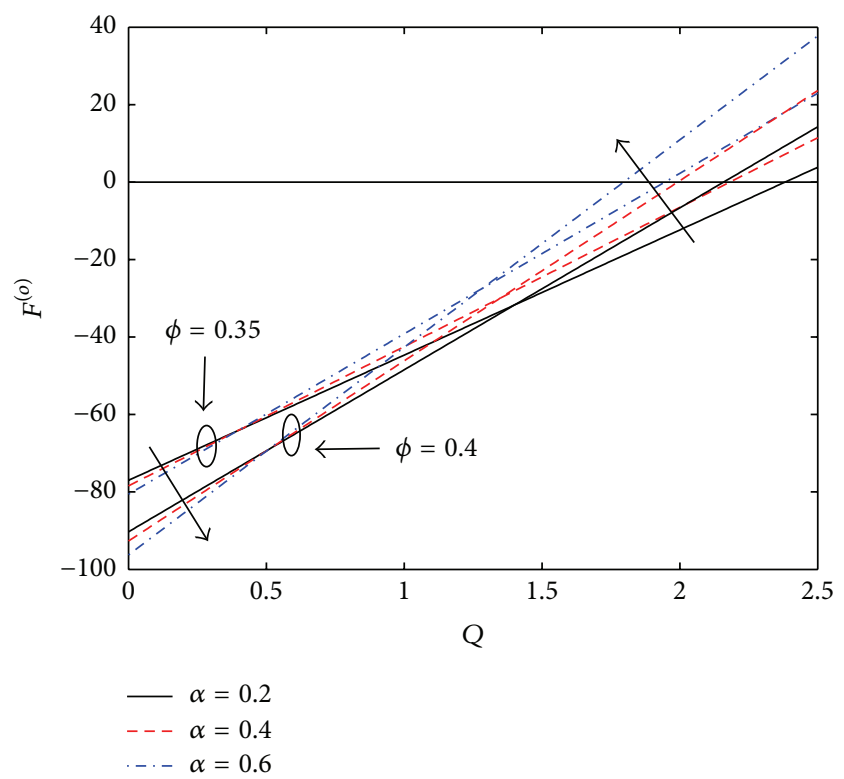

FIGURE 13: Frictional force on the outer tube $F^{(o)}$ versus the timeaveraged flow rate $Q$ for different values of the parameter $\alpha_{1}$ and amplitude ratio $\phi$ with $\mathrm{Gr}=3, \varepsilon=0.35, \beta=10, \lambda_{1}=1$, and $t=1$.

(4) $Q$ decreases with increasing $\phi$ and $\varepsilon$ in the freepumping and copumping regions.

(5) The frictional forces $F^{(i)}$ and $F^{(o)}$ have opposite behavior compared to the pressure rise $\Delta p$ versus the physical parameters.

\section{Appendix}

Consider

$$
\begin{aligned}
& a_{11}=\ln \left(\frac{\varepsilon}{r_{2}}\right) ; \\
& a_{12}=r_{2}^{2}-\varepsilon^{2} ; \\
& a_{13}=r_{2}^{2} \ln (\varepsilon) ; \\
& a_{14}=\varepsilon^{2} \ln \left(r_{2}\right) ; \\
& a_{15}=\frac{r_{2}^{2}}{a_{11}} ; \\
& a_{16}=\frac{\varepsilon_{2}^{2}}{a_{11}} ; \\
& a_{17}=\frac{r_{2}^{4}}{a_{11}} ; \\
& a_{18}=\frac{\varepsilon_{2}^{4}}{a_{11}} ;
\end{aligned}
$$




$$
\begin{aligned}
& a_{21}=\frac{a_{12}}{a_{11}} ; \\
& a_{22}=\frac{a_{13}}{a_{11}} ; \\
& a_{23}=\frac{a_{14}}{a_{11}} \text {; } \\
& a_{24}=\frac{a_{15}}{a_{11}} \text {; } \\
& a_{25}=\frac{a_{16}}{a_{11}} \text {; } \\
& a_{26}=a_{22}-a_{23} ; \\
& a_{27}=a_{18}-a_{17} \text {; } \\
& a_{28}=a_{15}^{2}-a_{16}^{2} \text {; } \\
& a_{29}=a_{13} a_{15}-a_{14} a_{16} \text {; } \\
& a_{31}=a_{16}+a_{24}+a_{26} \text {; } \\
& a_{32}=\frac{a_{26}}{a_{11}} ; \\
& a_{33}=a_{15} a_{26} \text {; } \\
& a_{34}=a_{16} a_{26} \text {; } \\
& a_{35}=a_{27}+a_{29} ; \\
& a_{36}=a_{33}-a_{34} ; \\
& a_{37}=2 a_{35}-a_{28} ; \\
& a_{38}=a_{23}+a_{32} ; \\
& a_{41}=\frac{4+\beta a_{25}}{a_{11}} ; \\
& a_{42}=\frac{4+\beta a_{12}}{a_{11}} ; \\
& a_{43}=4 \beta a_{36}-16 a_{39}+3 \beta a_{29} \text {; } \\
& a_{44}=a_{21}\left(r_{2}^{2} \ln \left(r_{2}\right)-\varepsilon^{2} \ln (\varepsilon)\right) ; \\
& a_{45}=a_{12}\left(2 a_{21}+a_{25}\right) \text {; } \\
& a_{46}=r_{2}^{4}-\varepsilon^{4} ; \\
& a_{47}=a_{11}\left(\varepsilon^{2} a_{41}-\beta r_{2}^{4} a_{24}\right) \text {; } \\
& a_{48}=a_{42} a_{45} \\
& a_{49}=a_{41} a_{45} ; \\
& a_{51}=a_{24} a_{45} ; \\
& a_{52}=a_{43} a_{12} \\
& a_{53}=r_{2}^{8}-\varepsilon^{8}
\end{aligned}
$$

$$
\begin{aligned}
a_{61} & =\frac{1}{8}\left[2 a_{44}+2 a_{45}+a_{46}\right] ; \\
a_{62} & =\frac{\mathrm{Gr}}{256}\left[8\left(a_{48}+a_{47}\right)+4 a_{52}+2 a_{49}\right. \\
& \left.+\beta\left(a_{53}-2 a_{51}\right)\right] .
\end{aligned}
$$

\section{Conflict of Interests}

The author declares that there is no conflict of interests regarding the publication of this paper.

\section{Acknowledgments}

The author is grateful to the referees and editors for their constructive suggestions.

\section{References}

[1] T. W. Latham, Fluid motions in a peristaltic pump [M.S. thesis], Massachusetts Institute of Technology, Cambridge, Mass, USA, 1966.

[2] M. R. Kaimal, "Peristaltic pumping of a newtonian fluid with particles suspended in it at low reynolds number under long wavelength approximations," Journal of Applied Mechanics, Transactions ASME, vol. 45, no. 1, pp. 32-36, 1978.

[3] A. H. Shapiro, M. Y. Jaffrin, and S. L. Weinberg, "Peristaltic pumping with long wavelengths at low reynolds number," Journal of Fluid Mechanics, vol. 37, no. 4, pp. 799-825, 1969.

[4] M. Mishra and A. R. Rao, "Peristaltic transport of a Newtonian fluid in an asymmetric channel," Zeitschrift für Angewandte Mathematik und Physik, vol. 54, no. 3, pp. 532-550, 2003.

[5] N. Ali, M. Sajid, Z. Abbas, and T. Javed, "Non-Newtonian fluid flow induced by peristaltic waves in a curved channel," European Journal of Mechanics, B/Fluids, vol. 29, no. 5, pp. 387-394, 2010.

[6] A. M. Abd-Alla, S. M. Abo-Dahab, and M. M. Albalawi, "Magnetic field and gravity effects on peristaltic transport of a jeffrey fluid in an asymmetric channel," Abstract and Applied Analysis, vol. 2014, Article ID 896121, 11 pages, 2014.

[7] H. Rachid and M. T. Ouazzani, "Interaction of pulsatile flow with peristaltic transport of a viscoelastic fluid: case of a Maxwell fluid," International Journal of Applied Mechanics, vol. 6, no. 5, Article ID 1450061, 2014.

[8] A. Medhavi, "Peristaltic pumping of a non-Newtonian fluid," Applications and Applied Mathematics, vol. 3, no. 1, pp. 137-148, 2008.

[9] J. B. Shukla and S. P. Gupta, "Peristaltic transport of a powerlaw fluid with variable consistency," Journal of Biomechanical Engineering, vol. 104, no. 3, pp. 182-186, 1982.

[10] K. S. Mekheimer, "Peristaltic transport of a newtonian fluid through a uniform and non-uniform annulus," Arabian Journal for Science and Engineering, vol. 30, no. 1, pp. 69-83, 2005.

[11] N. S. Akbar and S. Nadeem, "An analytical and numerical study of peristaltic transport of a Johnson-Segalman fluid in an endoscope," Chinese Physics B, vol. 22, no. 1, Article ID 014703, 2013.

[12] S. Z. A. Husseny, Y. Abd Elmaboud, and Kh. S. Mekheimer, "The flow separation of peristaltic transport for Maxwell fluid 
between two coaxial tubes," Abstract and Applied Analysis, vol. 2014, Article ID 269151, 17 pages, 2014.

[13] F. Mainardi and G. Spada, "Creep, relaxation and viscosity properties for basic fractional models in rheology," European Physical Journal: Special Topics, vol. 193, no. 1, pp. 133-160, 2011.

[14] H. Qi and H. Jin, "Unsteady rotating flows of a viscoelastic fluid with the fractional Maxwell model between coaxial cylinders," Acta Mechanica Sinica, vol. 22, no. 4, pp. 301-305, 2006.

[15] C. Fetecau, M. Athar, and C. Fetecau, "Unsteady flow of a generalized Maxwell fluid with fractional derivative due to a constantly accelerating plate," Computers and Mathematics with Applications, vol. 57, no. 4, pp. 596-603, 2009.

[16] M. Khan, S. H. Ali, and H. Qi, "Exact solutions for some oscillating flows of a second grade fluid with a fractional derivative model," Mathematical and Computer Modelling, vol. 49, no. 7-8, pp. 1519-1530, 2009.

[17] W. Tan and M. Xu, "Unsteady flows of a generalized second grade fluid with the fractional derivative model between two parallel plates," Acta Mechanica Sinica, vol. 20, no. 5, pp. 471476, 2004.

[18] Y. Liu, L. Zheng, and X. Zhang, "Unsteady MHD couette flow of a generalized oldroyd-B fluid with fractional derivative," Computers \& Mathematics with Applications, vol. 61, no. 2, pp. 443-450, 2011.

[19] Q. Haitao and X. Mingyu, "Some unsteady unidirectional flows of a generalized Oldroyd-B fluid with fractional derivative," Applied Mathematical Modelling, vol. 33, no. 11, pp. 4184-4191, 2009.

[20] M. Khan, A. Anjum, C. Fetecau, and H. Qi, "Exact solutions for some oscillating motions of a fractional Burgers' fluid," Mathematical and Computer Modelling, vol. 51, no. 5-6, pp. 682-692, 2010.

[21] D. Tripathi, "A mathematical model for the peristaltic flow of chyme movement in small intestine," Mathematical Biosciences, vol. 233, no. 2, pp. 90-97, 2011.

[22] D. Tripathi, "Peristaltic flow of a fractional second grade fluid through a cylindrical tube," Thermal Science, vol. 15, no. 2, pp. S167-S173, 2011.

[23] V. P. Rathod and M. M. Channakote, "Interaction of heat transfer and peristaltic pumping of fractional second grade fluid through a vertical cylindrical tube," Thermal Science, vol. 18, no. 4, pp. 1109-1118, 2014.

[24] D. Tripathi, S. K. Pandey, and S. Das, "Peristaltic flow of viscoelastic fluid with fractional Maxwell model through a channel," Applied Mathematics and Computation, vol. 215, no. 10, pp. 3645-3654, 2010.

[25] D. Tripathi, P. K. Gupta, and S. Das, "Influence of slip condition on peristaltic transport of a viscoelastic fluid with fractional Burger's model," Thermal Science, vol. 15, no. 2, pp. 501-515, 2011.

[26] M. Khan, S. Hyder Ali, and H. Qi, "On accelerated flows of a viscoelastic fluid with the fractional Burgers' model," Nonlinear Analysis: Real World Applications, vol. 10, no. 4, pp. 2286-2296, 2009.

[27] A. M. Siddiqui, M. A. Rana, and N. Ahmed, "Magnetohydrodynamics flow of a Burgers' fluid in an orthogonal rheometer," Applied Mathematical Modelling, vol. 34, no. 10, pp. 2881-2892, 2010.

[28] S. H. A. M. Shah, "Some helical flows of a Burgers' fluid with fractional derivative," Meccanica, vol. 45, no. 2, pp. 143-151, 2010.

[29] C. Vasudev, U. Rajeswara Rao, and G. Prabhakara Rao, "Peristaltic flow of a Newtonian fluid through a porous medium in a vertical tube under the effect of a magnetic field," International Journal of Current Science, vol. 1, no. 3, pp. 105-110, 2011.

[30] T. Hayat and F. M. Abbasi, "Peristaltic mechanism in an asymmetric channel with heat transfer," Mathematical and Computational Applications, vol. 15, no. 4, pp. 621-637, 2010.

[31] T. Hayat, S. Hina, and A. A. Hendi, "Peristaltic motion of powerlaw fluid with heat and mass transfer," Chinese Physics Letters, vol. 28, no. 8, Article ID 084707, 2011.

[32] I. Podlubny, Fractional Differential Equations, Accademic Press, San Diego, Calif, USA, 1999. 


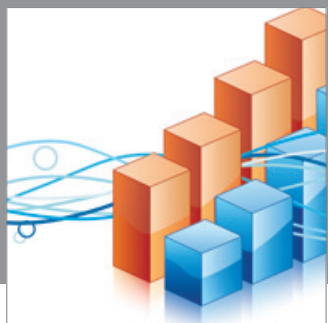

Advances in

Operations Research

mansans

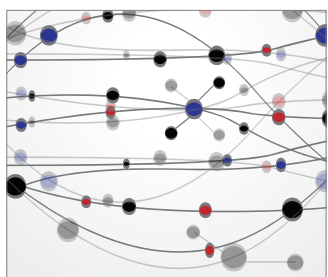

The Scientific World Journal
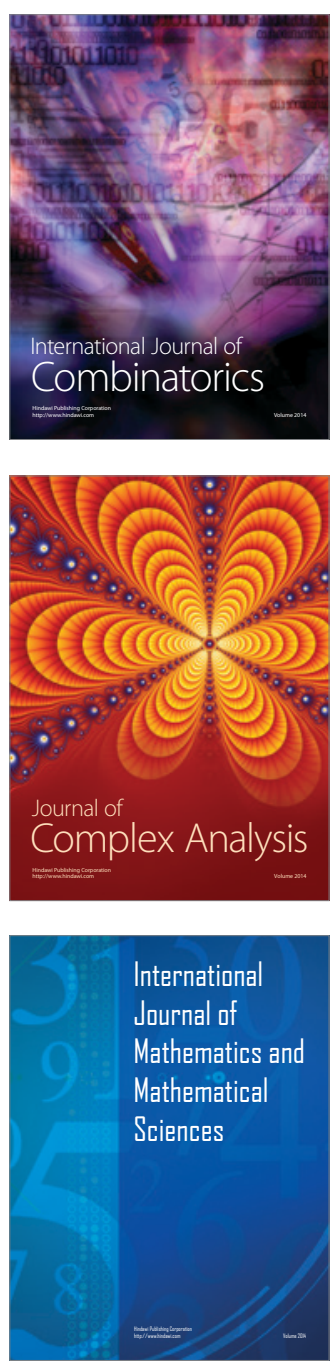
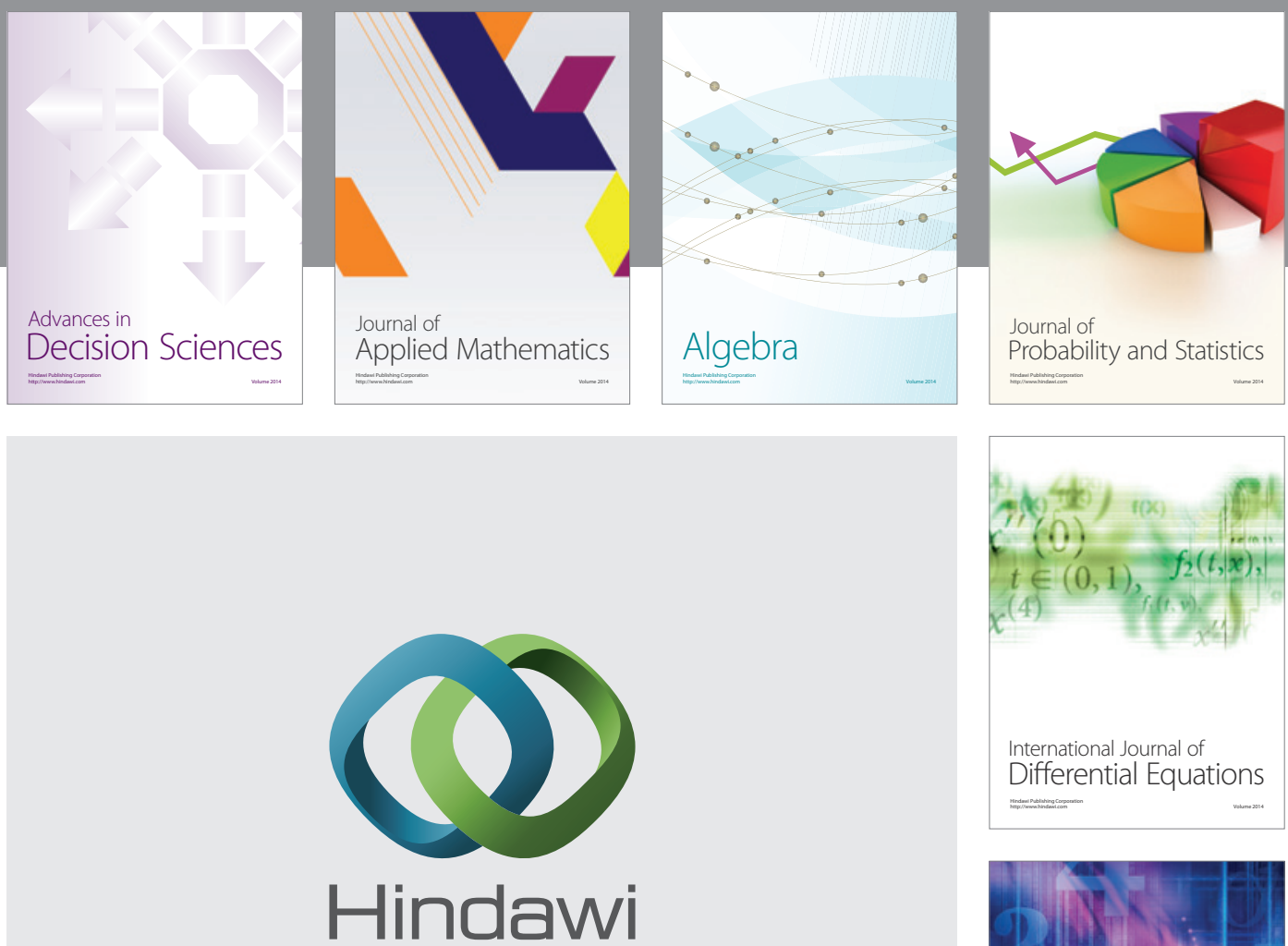

Submit your manuscripts at http://www.hindawi.com
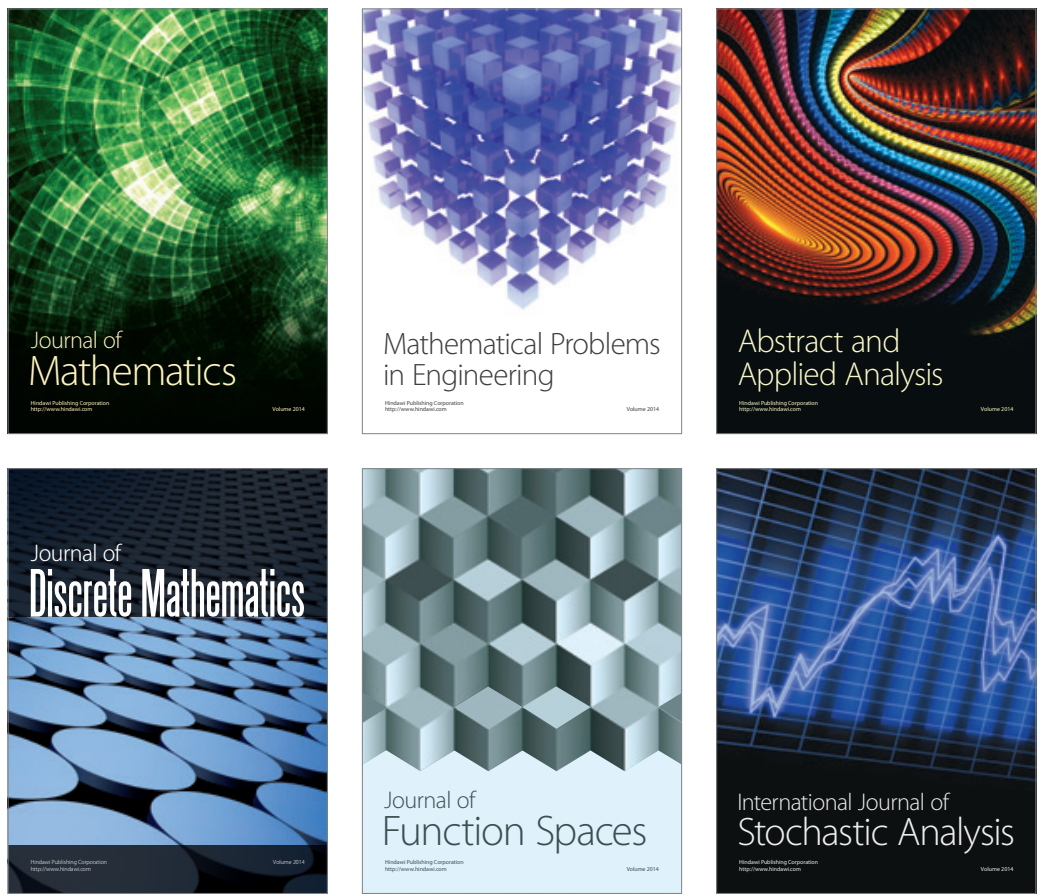

Journal of

Function Spaces

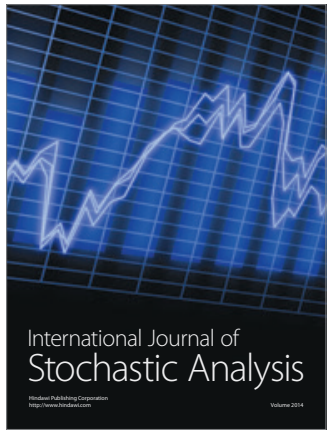

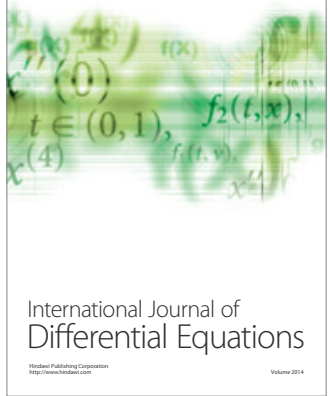
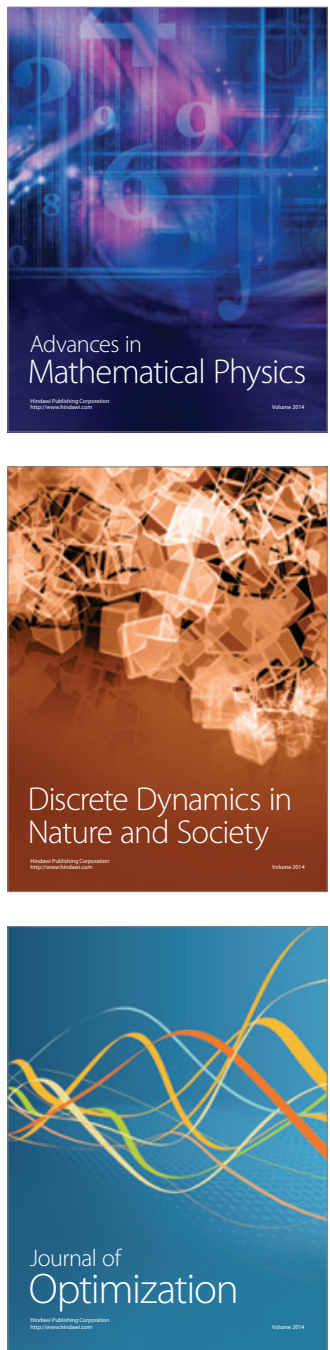